\title{
CLASSICAL HEREDO-FAMILIAL DYSTROPHIES OF THE CORNEA
}

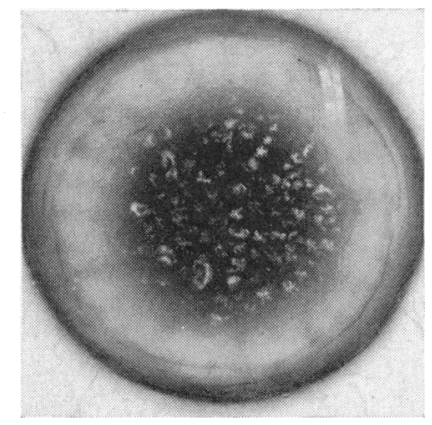

Fig. 1.-Granular dystrophy.

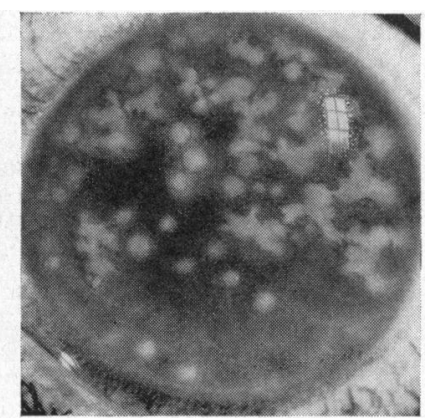

Fig. 2.-Macular dystrophy.

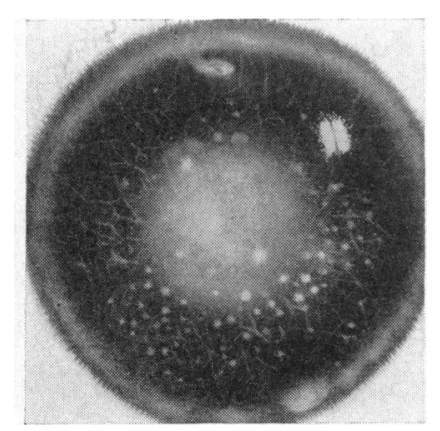

FIG. 3.-Lattice dystrophy.

\section{Granular Dystrophy (Type I of Groenouw)}

Dominant transmission.

Appears in first decade of life.

Presents as dots and radial lines which progress to discrete greyish opacities of variable form.

Commences beneath Bowman's membrane, later becomes deeper.

Occupies the central two-thirds of the cornea and spares the periphery.

The corneal surface is smooth in the young and rough in older patients.

Corneal sensation is normal or diminished.

Decrease in vision is not as marked as in other dystrophies; the subject may be able to work until old age.

\section{Macular Dystrophy (Type II of Groenouw)}

Recessive transmission.

Appears in first or second decades of life.

Presents as a slight clouding of the entire corneal surface which increases in density. Large and small spots with ill-defined borders appear in diffuse opacity which involves the whole thickness of the stroma.

The periphery of the cornea is involved.

Corneal surface is irregular.

Corneal sensation is normal or diminished.

Vision is severely affected between 20 to 40 years of age.

\section{Lattice Dystrophy}

Dominant transmission.

Appears towards the end of the first decade of life.

Presents as striae and dots forming a lattice pattern which affects much of the stromal depth.

Corneal surface is irregular.

Corneal sensation is diminished in the centre, and normal at the periphery.

Vision is usually severely affected by 40 years of age.

Inflammatory attacks appear early and are characteristic.

General Reference

WAARDENBURG, P. J., Franceschetti, A., and KLein, D. (1961). “Genetics and Ophthalmology" vol. 1, p. 462. Van Gorcum, Assen, Netherlands. 


\section{PATHOLOGY}

\section{Granular Dystrophy}

Non-specific changes in the epithelium and Bowman's membrane. The hyaline, granulated stromal lesions stain intensely with Masson's trichrome, and are not birefringent. They consist of focal areas of hyaline degeneration presenting as coarse and fine granules frequently aggregated into rectangles. Electron microscopy reveals vacuolation of corneal stromal cells (fibroblasts) which contain a material of variable density.

Electron microscopic evidence points to granular dystrophy being a primary intracellular degeneration of corneal stromal cells progressing to extracellular deposits in the corneal stroma of degenerative material and stromal cell remnants.

SoRnson, E. T. (1965). Amer. J. Ophthal., 59, 1001.

\section{Macular Dystrophy}

Non-specific changes in the epithelium and Bowman's membrane. With PAS, alcian blue, or colloidal iron stains conspicuous alterations are seen throughout the stroma. These consist of non-hyalinized, granulated material within corneal fibroblasts and in the surrounding collagen. Electron microscopy reveals vacuoles within the cytoplasm of the fibroblasts.

Electron microscopic and histochemical evidence points to macular dystrophy being a metabolic disorder restricted to corneal fibroblasts, and characterized by the intracellular storage and extracellular migration and accumulation of an acid mucopolysaccharide material.

Klintworth, G. K., and Vogel, F. S. (1964). Amer. J. Path., 45, 565.

\section{Lattice Dystrophy}

Non-specific changes in the epithelium and Bowman's membrane. The stromal lesions have a hyaline and fibrillar appearance, and often a fusiform shape. They stain intensely with Masson's trichrome and PAS, and are usually more birefringent than the surrounding stroma.

Electron microscopy shows the lesions to consist of two distinct parts: (1) disoriented filaments about one-third the diameter of normal stromal collagen fibrils, and (2) modified stromal lamellae with fibrils of normal diameter but increased density. The first are non-birefringent, the second are birefringent.

Electron microscopic evidence points to lattice dystrophy as a "fibrillary degeneration" of corneal stromal collagen.

MCTigue, J. W., and Fine, B. S. (1964). Invest. Ophthal., 3, 355.

General Reference

Jones, S. T., and Zimmerman, L. E. (1961). Amer. J. Ophthal., 51, 394.

Illustrations

INSTITUTE OF OPHTHALMOLOGY.

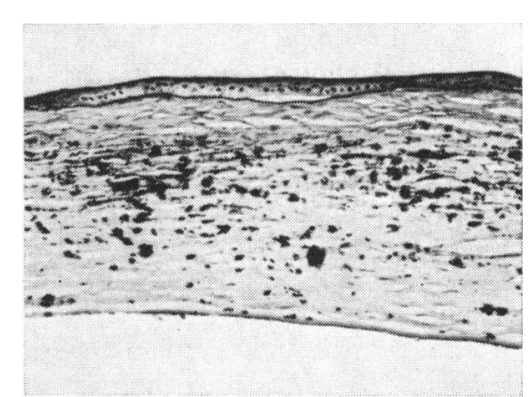

Fig. 4.-Granular dystrophy. Masson's trichrome. $\times 60$.

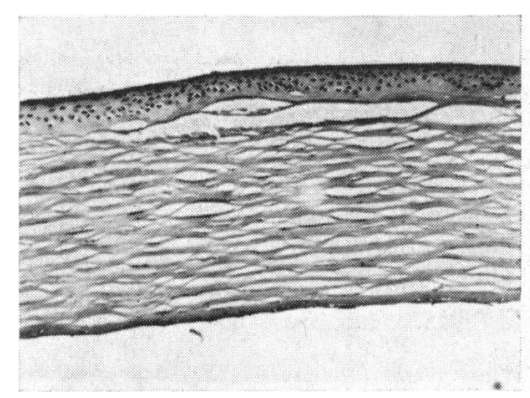

Fig. 5.-Macular dystrophy. Alcian blue. $\times 60$.

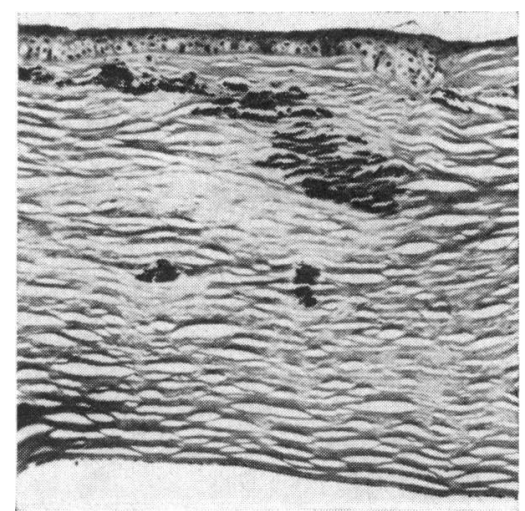

FIG. 6.-Lattice dystrophy. Masson's trichrome. $\times 60$. 